\title{
Clinical Vignettes \\ Drug-Induced Liver Injury in the Setting of Glycogenic Hepatopathy
}

\author{
Valmiki Maharaj, $M D^{7}$, Matthew Fitz, $M D^{7}$, and Xianzdong Ding, $M D P h D^{2}$ \\ 'Department of Medicine, Loyola University Stritch School of Medicine, Maywood, IL, USA; ${ }^{2}$ Department of Pathology, Loyola University Stritch \\ School of Medicine, Maywood, IL, USA.
}

Glycogenic hepatopathy $(\mathrm{GH})$ is an underdiagnosed complication of uncontrolled type 1 diabetes mellitus (T1DM). It appears as an acute relapsing hepatitis with reversible transaminase elevations secondary to excessive hepatic glycogen accumulation. Patients are often asymptomatic but can present with abdominal pain, nausea and vomiting. Physical examination shows hepatomegaly without splenomegaly. GH is diagnosed by biopsy as it is clinically indistinguishable from non-alcoholic fatty liver disease (NAFLD), a more common cause of hepatic dysfunction in diabetics. Here we describe a case of $\mathrm{GH}$ in a patient with uncontrolled type 1 diabetes whose clinical course was complicated by drug-induced liver injury. The patient initially presented with diabetic ketoacidosis and had a mild transaminitis, thought to be due to NAFLD. She developed profound transaminase elevations while receiving treatment with newer antipsychotic medications for her bipolar disorder. Liver biopsy showed evidence of resolving glycogenic hepatopathy with signs of druginduced liver injury. This case report reviews the pathology and pathogenesis of $\mathrm{GH}$ and reminds the clinician to keep GH within the differential diagnosis for severe transaminitis in a patient with type 1 diabetes mellitus.

J Gen Intern Med 32(6):714-7

DOI: $10.1007 / \mathrm{s} 11606-017-3996-\mathrm{Z}$

(c) Society of General Internal Medicine 2017

\section{INTRODUCTION}

Glycogenic hepatopathy (GH) is an underdiagnosed complication of uncontrolled type 1 diabetes mellitus (T1DM). ${ }^{1}$ It appears as an acute relapsing hepatitis secondary to excessive hepatic glycogen accumulation. Fortunately, the transaminase elevations are reversible. Although most patients are asymptomatic, they can present with abdominal pain, nausea and vomiting. Physical examination shows hepatomegaly without splenomegaly. Perihepatic or diffuse ascites is possible. GH is diagnosed by biopsy, as it is clinically difficult to distinguish from non-alcoholic fatty liver disease (NAFLD), a much more common cause of hepatic dysfunction in diabetics. Here we describe a case of GH in a patient with uncontrolled T1DM whose clinical course was complicated by drug-induced liver injury.

Received March 16, 2016

Revised July 11, 2016

Accepted January 13, 2017

Published online February 21, 2017

\section{CASE REPORT}

A 23-year-old female, diagnosed with T1DM at age 6, was admitted to the medical intensive care unit for diabetic ketoacidosis (DKA). She had several prior admissions for DKA because of poorly controlled T1DM (HbA1c 12.3\%). Her outpatient regimen consisted of insulin glargine and mealtime insulin aspart. Upon presentation, her serum glucose was $1318 \mathrm{mg} / \mathrm{dl}$ (reference range 65-110 $\mathrm{mg} / \mathrm{dl}$ ), anion gap 29 $\mathrm{mEq} / 1(8-12 \mathrm{mEq} / \mathrm{l})$ and $\mathrm{pH} 7.27$ (7.35-7.45). She was treated with intravenous fluids and insulin. Her chief complaint was abdominal pain, and her physical examination noted right upper quadrant tenderness with hepatomegaly $12 \mathrm{~cm}$ below the costal margin in the mid-clavicular line. Her liver function tests (LFTs) were elevated: aspartate aminotransferase (AST) 127 IU/1 (5-30 IU/1), alanine aminotransferase (ALT) 199 IU/1 (5-30 IU/1), alkaline phosphatase (ALP) 160 IU/1 (50-100 IU/ 1) and total bilirubin $2.7 \mathrm{mg} / \mathrm{dl}(0-1.9 \mathrm{mg} / \mathrm{dl})$. These laboratory tests had been normal during her last hospital admission 4 months prior but did have slight elevations during previous admissions at other hospitals. Magnetic resonance imaging (MRI) and computed tomography (CT) showed massive hepatomegaly (measuring $26.0 \mathrm{~cm}$ ) without steatosis or focal lesions, similar to scans 2 years earlier. Acute viral hepatitis serologies for A, B and C as well as serologies for cytomegalovirus and Epstein-Barr virus were negative. Autoantibodies including anti-nuclear antibody (ANA), smooth muscle antibodies (ASMA) and mitochondrial antibodies (AMA) were also negative. Tests for ceruloplasmin (Wilson's disease), alpha-one antitrypsin and HFE gene (hereditary hemochromatosis) were negative as well. Acetaminophen, salicylates and blood alcohol levels were undetectable, and a urine drug screen was negative. The presumed cause was nonalcoholic fatty liver disease.

Her hospital course was complicated by uncontrolled bipolar disease. Her outpatient medications on admission consisted of aripiprazole, escitalopram and lorazepam, though notably the patient had been tried on several different regimens previously. During her inpatient stay, asenapine, an atypical antipsychotic, was recommended by the consulting psychiatry team to control her symptomatology. Thirty hours after administration, the patient had an AST level of 3600 IU/1. Hepatology was consulted, and serologies were repeated. The patient's vital signs remained stable, and Doppler abdominal ultrasound showed patent vasculature. Because druginduced liver injury was suspected, asenapine was 
discontinued, and her transaminase levels began to trend back to her admission levels. Paliperidone, another atypical antipsychotic, was administered, causing similar transaminitis without a change in her clinical status. Again, the medication was discontinued, and her transaminitis again trended back to her prior values on admission (AST 120, ALT 190, ALP 170).

The clinical differential diagnosis for her liver injury pattern included NAFLD, drug-induced liver injury and autoimmune hepatitis, among others; therefore, a liver biopsy was performed. Hematoxylin and eosin (H\&E) stain revealed patchy hepatocyte enlargement with markedly increased cytoplasmic volume and a lightly stained, finely rarefied cytoplasmic content (Fig. 1). PAS stain highlighted glycogen granules in the swelled hepatocyte cytoplasm, which disappeared on diastase treatment (Fig. 2). These findings were consistent with glycogenic hepatopathy given the patient's clinical history and presentation. Numerous glycogenated nuclei were present in the hepatocytes. There was no significant portal inflammation or significant fibrosis to suggest cirrhosis. No significant macrovesicular steatosis (NAFLD), Mallory-Denk body formation or satellitosis (alcoholic liver disease), or iron overload (hemochromatosis) were identified. In addition, the biopsy showed histological features of concurrent mild druginduced liver injury: "rare hepatocyte dropouts replaced by small clusters of lymphocytes," "rare pigmented Kupffer cells" and "eosinophil infiltrates" (Fig. 1).

The patient was discharged on stable doses of insulin for diabetes and buspirone and lithium for her bipolar disorder. Her LFTs returned to normal 3 weeks after admission. Since her initial hospitalization, the patient was readmitted several more times for diabetic ketoacidosis and abdominal pain. During these admissions, she presented with mild transaminitis on admission that peaked and resolved over the course of her hospitalizations, thought to be due to DKA and her underlying GH. No other etiologies have been discovered to explain the transaminitis. Her $\mathrm{HbAl} \mathrm{c}$ has since improved to $8.1 \%$ from $12.3 \%$. Subsequently, her hepatomegaly has also decreased from $3640 \mathrm{~cm}^{3}$ to $2700 \mathrm{~cm}^{3}$.

\section{DISCUSSION}

In T1DM the leading causes of liver injury are NAFLD and autoimmune hepatitis. NAFLD is common because of the associated comorbidities, and autoimmune hepatitis is seen in association with other autoimmune diseases, such as thyroid and adrenal disorders. It is also important to consider the numerous enzyme deficiencies that cause the glycogen storage diseases. Diagnosing the root cause of the transaminitis is paramount because the treatments differ. Fortunately, GH and the associated transaminitis resolve with proper diabetes treatment-adequate insulin therapy and maintaining euglycemia - although normalization of laboratory values can take months. GH does not cause hepatic complications, such as cirrhosis and hepatocellular carcinoma, seen in other chronic long-standing liver disorders. Although GH is typically distinguished from NAFLD on biopsy, subtle differences on imaging can exist. GH can appear as a hyperdense (75 HU) liver on CT and can point toward GH, especially if contrast is not used. ${ }^{2}$ However, other reported etiologies of a hyperdense liver include iron deposition, chronic arsenic poisoning, colloidal gold therapy, thallium poisoning and Wilson's disease. Another distinction is that GH is isointense between phases on gradient dual-echo MRI, whereas NAFLD is low intensity in phase and high intensity out of phase. ${ }^{3,4}$ This radiological

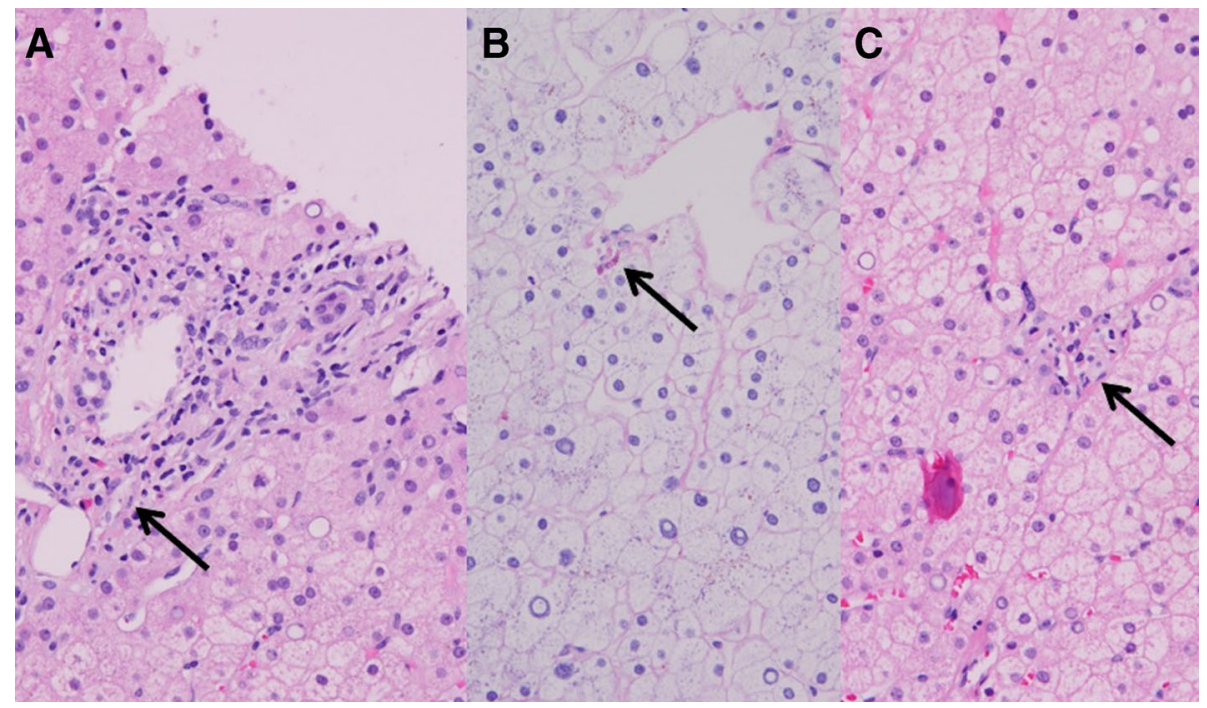

Figure 1 Hematoxylin and eosin $(H \& E)$ stain revealed patchy hepatocyte enlargement with markedly increased cytoplasmic volume and lightly stained, finely rarefied cytoplasmic content. Panel A: Portal inflammation with eosinophil and lymphocyte infiltration in portal tracts (arrow). Panel B: Pigmented Kupffer cells highlighted by a PAS-d stain (arrow). Panel C: Hepatocyte dropouts replaced by a cluster of lymphocytes (arrow) 


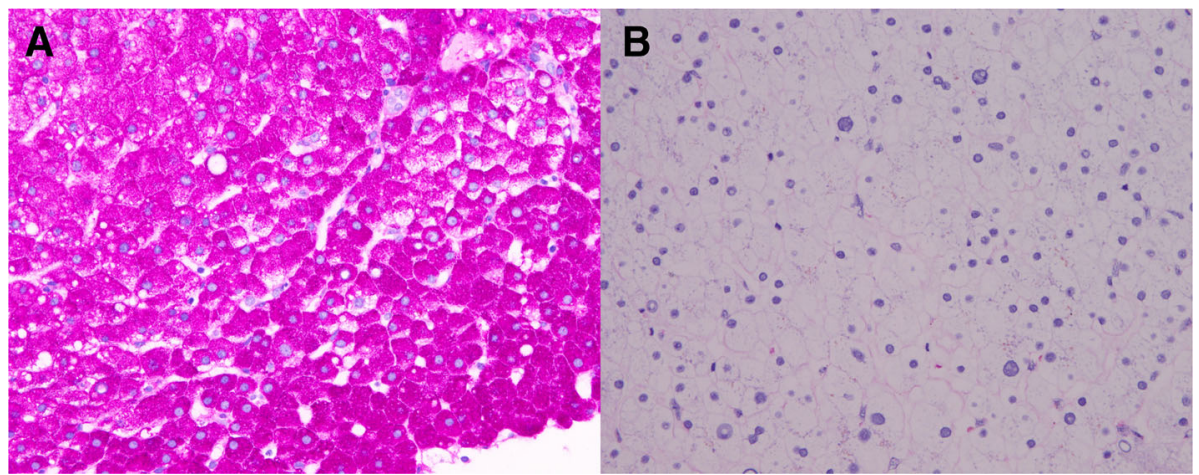

Figure 2 PAS stain highlighted glycogen granules in the swollen hepatocyte cytoplasm (A), which disappeared on diastase treatment (B)

distinction could not be made in our patient because the appropriate imaging sequences were not performed.

GH was first described by Mauriac in 1930 (shortly after insulin became medicalized) as part of Mauriac's syndrome, seen in children with poor glycemic control. Notable signs were Cushingoid features, growth impairment and pubertal delay, hepatomegaly and hyperlipidemia. Liver biopsies in these cases showed glycogen accumulation. Since then approximately 50 cases of adults have been described in the literature without the associated syndromic features. ${ }^{5}$

The essential pathophysiology of GH is the effect of large fluctuations in glucose and insulin levels on hepatocytes. ${ }^{6}$ In hepatocytes, unlike other cells, glucose undergoes insulinindependent facilitated diffusion into the cell. Glucose then undergoes glycogenesis via several enzymatic steps that can be accelerated by high glucose levels and high insulin requirements, such as during treatment of DKA. Glycogenolysis is activated by glucagon and epinephrine during periods of relative hypoglycemia. It is thought that the severe fluctuations between hyperglycemia and relative hypoglycemia perpetuate a cycle of gluconeogenesis and glycogenesis, exacerbating and worsening glycogen accumulation. Histology shows pale, enlarged hepatocytes that stain PAS positive because of glycogen accumulation. ${ }^{7}$ Hepatic sinusoids can be compressed because of congestion, though signs of necrosis or cholestasis are typically not seen. Steatosis and fibrosis may be present in a minority of cases but is usually minimal to mild.

One hypothesis for the accompanying transaminitis is cell enzyme "leakage" due to the marked glycogen accumulation rather than cellular necrosis. Transient elevations in the transaminases seen after insulin treatment for ketoacidosis support this hypothesis, consistent with our patient's clinical history. To interrupt the cycle, a relatively stable period of euglycemia with minimal insulin usage is needed. With routine diabetes care and treatment, GH can resolve as quickly as it develops. ${ }^{8}$ Cases involving pancreas transplant reversing GH have also been described. ${ }^{9}$

As of this writing, no cases of GH with concomitant druginduced liver injury were found on a Pubmed literature search. Asenapine and paliperidone are atypical antipsychotics used for treatment of schizophrenia and bipolar disorder. ${ }^{10,11}$ They are primarily serotonergic antagonists metabolized by the liver cytochrome P450 isoenzymes. Asenapine is contraindicated in patients with severe hepatic impairment (Child-Pugh C), although no dose adjustment is needed for mild impairment. ${ }^{12}$ Paliperidone has no necessary dose adjustments for those with hepatic dysfunction. ${ }^{13}$ Potential complications of each include hypersensitivity reactions, neuroleptic malignant syndrome, as well as hyperglycemia and diabetes mellitus. In short-term trials of adult bipolar patients, asenapine increased mean ALT 8.9 units/l, and $2.5 \%$ of patients had elevated levels greater than three times the upper limit of normal. ${ }^{12}$ Increased serum transaminase levels can be seen in up to $2 \%$ of patients taking paliperidone. ${ }^{13}$

Currently it is not known whether patients with GH are predisposed to drug-induced liver injury or at risk for altered hepatic synthetic or metabolic function. Poor liver synthetic and metabolic function has not been described in the literature for GH patients. Synthetic function is typically preserved, though it is not uncommon to have hypoalbuminemia. ${ }^{6}$ The leading hypothesis is that the patient's underlying $\mathrm{GH}$ predisposed her to significant liver injury with the atypical antipsychotic medications. Additionally, her drug-induced liver injury could have been exacerbated by decreased metabolization of the antipsychotics because of her GH. Although the atypical antipsychotic medications have less risk of liver injury than typical antipsychotic medications, they could be responsible for increased hyperglycemia, complicating her disease further. More data will be beneficial as the atypical antipsychotic medications become more prevalent in use.

\section{CONCLUSION}

$\mathrm{GH}$ is an underdiagnosed liver condition that is predominantly seen in uncontrolled T1DM. It is clinically indistinguishable from NAFLD, and biopsy is needed for diagnosis. Essential treatment is maintaining euglycemia at which symptoms and signs should resolve. With adequate treatment, patients are not at risk to develop complications of chronic liver injury and dysfunction and should return to normal hepatic function. Although it has not been described previously whether these 
patients are more prone to hepatic injury from the prescribed drugs, the initiation and cessation of two atypical antipsychotic medications followed by profound transaminitis suggest caution when using these medications in patients with $\mathrm{GH}$ specifically. The use of these medications warrants further investigation in patients with TIDM in general.

Corresponding Author: Valmiki Maharaj, MD; Department of MedicineLoyola University Stritch School of Medicine, Room 7612, 2160 S 1st Avenue, Maywood, IL 60153, USA (e-mail: valmiki. maharaj@lumc.edu).

\section{Compliance with Ethical Standards:}

Conflict of Interest: The authors declare that they do not have a conflict of interest.

\section{REFERENCES}

1. Messeri S, Messerini L, Vizzutti F, Laffi G, Marra F. Glycogenic hepatopathy associated with type 1 diabetes mellitus as a cause of recurrent liver damage. Ann Hepatol. 2012;11(4):554-8.

2. Sweetser S, Kraichely RE. The bright liver of glycogenic hepatopathy. Hepatology. 2010;51(2):711-2.
3. Murata F, Horie I, Ando T, et al. A case of glycogenic hepatopathy developed in a patient with new-onset fulminant type 1 diabetes: the role of image modalities in diagnosing hepatic glycogen deposition including gradient-dual-echo MRI. Endocr J. 2012;59(8):669-76.

4. Saikusa M, Yatsuga S, Tonan T, Koga Y. Glycogenic hepatopathy and non-alcoholic fatty liver disease in type 1 diabetes patients. Pediatr Int: Off J Japan Pediatr Soc. 2013;55(6):806-7.

5. van den Brand M, Elving LD, Drenth JP, van Krieken JH. Glycogenic hepatopathy: a rare cause of elevated serum transaminases in diabetes mellitus. Neth J Med. 2009;67(11):394-6.

6. Chatila R, West AB. Hepatomegaly and abnormal liver tests due to glycogenosis in adults with diabetes. Medicine. 1996;75(6):327-33.

7. Torbenson M, Chen YY, Brunt E, et al. Glycogenic hepatopathy: An under recognized hepatic complication of diabetes mellitus. Am J Surg Pathol. 2006;30(4):508-13.

8. Saadi T. Glycogenic hepatopathy: A rare disease that can appear and resolve rapidly in parallel with glycemic control. Isr Med Assoc J. 2012;14(4):269-70.

9. Fridell JA, Saxena R, Chalasani NP, Goggins WC, Powelson JA Cummings OW. Complete reversal of glycogen hepatopathy with pancreas transplantation: Two cases. Transplantation. 2012;83(1):84-6.

10. Actavis. Saphris: Full prescribing information. 2015

11. Janssen Pharmaceuticals. Invega: Full prescribing information. 2014.

12. Peeters $\mathbf{P}$, Bockbrader H, Spaans E, et al. Asenapine pharmacokinetics in hepatic and renal impairment. Clin Pharmacokinet. 2011;50(7):47181.

13. Amatniek J, Canuso CM, Deutsch SI, et al. Safety of paliperidone extended-release in patients with schizophrenia or schizoaffective disorder and hepatic disease. Clin Schizophrenia Relat Psychoses. 2014;8(1):8-20. 